\title{
Development of Simulating Wave System (SWS) for a Hospitable Sleeping Environment with Artificial Vibration
}

\author{
Ami Furuzono $^{a}$, Kenji Moriya ${ }^{\mathrm{b}}$, Kazuhiro Koshic $^{\mathrm{c}}, \mathrm{Keiji}_{\text {Matsumoto }}{ }^{\mathrm{a}^{*}}$ \\ ${ }^{a}$ National Institute of Technology, Kitakyushu College 5-20-1 Shii, Kokuraminami, \\ Kitakyushu City, Fukuoka, 802-0985, Japan \\ ${ }^{b}$ National Institute of Technology, Hakodate College, 14-1, Tokura-cho, Hakodate City, 042-8501, Japan \\ ${ }^{c}$ National Institute of Technology, Kumamoto College, 14-1, Suya, Koshi City, 2659-2, Japan \\ *Corresponding Author:kmatsu@kct.ac.jp
}

\begin{abstract}
In this study, we propose a simulating wave system (SWS) that provides a comfortable sleep environment to release stress. This system realizes floating-feeling as if it feels like floating on the water surface by automatically swinging the seat back and forth, left and right. First, this paper describes the mechanical structure and control method on the developed prototype SWS. Since we assess the comfort levels of SWS with subjective and objective evaluations, we describe these criteria in detail. For objective evaluation, four types of criteria, such as LF/HF, ellipse area, $\mathrm{SD} 1 / \mathrm{SD} 2$, and $\mathrm{SD} 1$, are adopted. $\mathrm{LF} / \mathrm{HF}$ calculated from the Fourier transformed subject's heart rate variability (HRV) and SD1/SD2 meaning the ratio of the major axis to the minor axis on a Lorenz plot are the values indicating sympathetic nerve activity. Ellipse areas on the Lorenz plot and minor axis SD1 are used as the value of parasympathetic nerve activity. For the subjective evaluation, a visual analog scale(VAS) is adopted that is a method of assessing sensation on a horizontal straight line ranging from 0 to 100 percent. This paper reports the research results of the proposed SWS on the above items.
\end{abstract}

Keywords: comfort, Lorentz plot, heart rate variability

\section{Introduction}

Sleep is the natural rest-activity observed in most creatures and is indispensable for life. It is known that sleep plays an important role in our life activities such as maintaining performance and body environment, the formation of learning and memory, and body growth, etc. [1].
A growth hormone, which strengthens the immune system by secreted by the pituitary gland during sleep, has also drawn attention in recent years [2,3]. However, according to a survey on national health conducted by the Ministry of Health, Labor and Welfare in 2017, only about 20 percent of people had no sleep problem. This result indicates that many people have dissatisfactions or difficulties with their sleep to some extent [4]. Poor sleep adversely affects human health such as a decline in cognitive ability, increased fatigue, and decreased vital energy, etc. [2,3]. Reynolds et al. reported that sleep deprivation causes an increase in absence rate, pointing out that the problems related to sleep even affect productivity in the workplace [5]. Creating a comfortable indoor environment is of great significance since it is suggested that addressing sleep problems appropriately great contributes to social health [6]. In a typical living environment, comfort is created through light, sound, and temperature, while the vibrational element is overlooked as a disagreeable element [7]. However, the category of vibration includes both unpleasant and pleasant elements. An infant's cradle has wonderful features that provide comfort. See-saws, swings, and hammocks are practical examples of products that use swinging to release stress. Moreover, comfortable vibration in a car makes people lethargic. The active rocking chair has been developed that has employed this oscillation characteristic successfully [8,9]. In this study, although the comfort of a rocking chair that is intrinsically controlled using a person's heart rate variability (HRV) is evaluated using a questionnaire, it is not directly related to the sleep environment and didn't assess comfort level on objective evaluations. In terms of controlling the bed automatically, the study of attitude control to protect an 
infant cradle mounted in a car from the impact of a car crash is also conducted $[10,11]$. However, this study is not the system producing a comfortable environment for sleep.

In this study, we propose a simulating wave system (SWS) that produces a comfortable environment to release stresses. This system realizes floating-feeling as if it feels like floating on the water surface by automatically swinging the seat back and forth, left and right. Proposed SWS is expected to provide a wonderful sleep environment because it gives people a comfortable floating feeling. If its comfort leads to reducing sleep latency and improving sleep quality, this system might be thought of as one of the solutions to contribute to health maintenance and preventative medicine. This time, we develop a prototype of SWS and evaluate it through subjective and objective evaluation methods. The subject's HRV is analyzed with four values of objective evaluation methods and a visual analog scale (VAS) is adopted as a subjective evaluation method. This paper describes the research results of SWS with the above items.

\section{Method}

\subsection{Mechanical structure}

Figure.1 and Figure.2 show the appearance of the system we proposed. The seat with an angle of 135 degrees is fixed on a constructed control unit in size of $550 \mathrm{~mm}$ long $\times 600 \mathrm{~mm}$ wide $\times 730 \mathrm{~mm}$ high. The seat base supported at the center by an iron bar of $25 \mathrm{~mm}$ diameter is constructed with a pair of bearing so as to be able to operate like a balancing toy. Two 750 watt servo motors are used to drive the seat and these motors have harmonic gear with a gear ratio of 300: 1 on the motor's head to increase driving torque. The seat base has mechanical linkage structures connected to the harmonic gear through the iron shafts so as to be controlled by the pair of servo motors. Various motions for the seat are possible to realize by controlling the pair of motors in any rotational direction. Motions of the seat from the front view are shown in Fig.3 and Fig.4. When both motors rotate $\theta$ degree to $\mathrm{CW}$ direction simultaneously, the seat leans to rightward. In the same way, the seat leans leftward when the motors simultaneously rotate any degree to the $\mathrm{CCW}$ direction. The left side motor rotates to the $\mathrm{CCW}$ direction and the right side motor rotates to the $\mathrm{CW}$ direction, then the seat leans forward. In the opposite way, the left side motor rotates to the $\mathrm{CW}$ direction and the right side motor rotates to the $\mathrm{CCW}$ direction, then the seat leans backward. As mentioned above, it is possible to realize 3D oscillation of the floating feeling by controlling the motors in various directions.

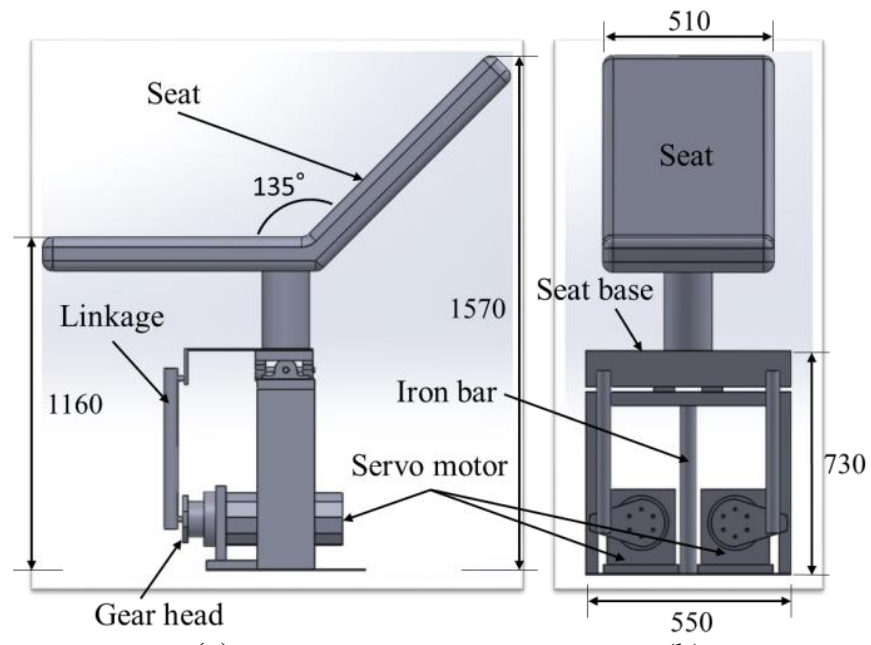

(a)

(b)

Fig.1 SWS designed with 3D CAD (a) Side view (b) Front view

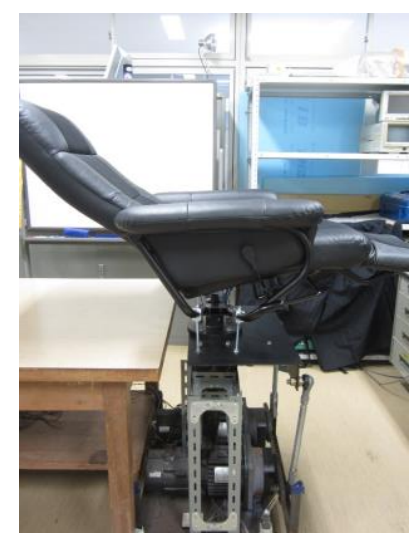

(a) (b)
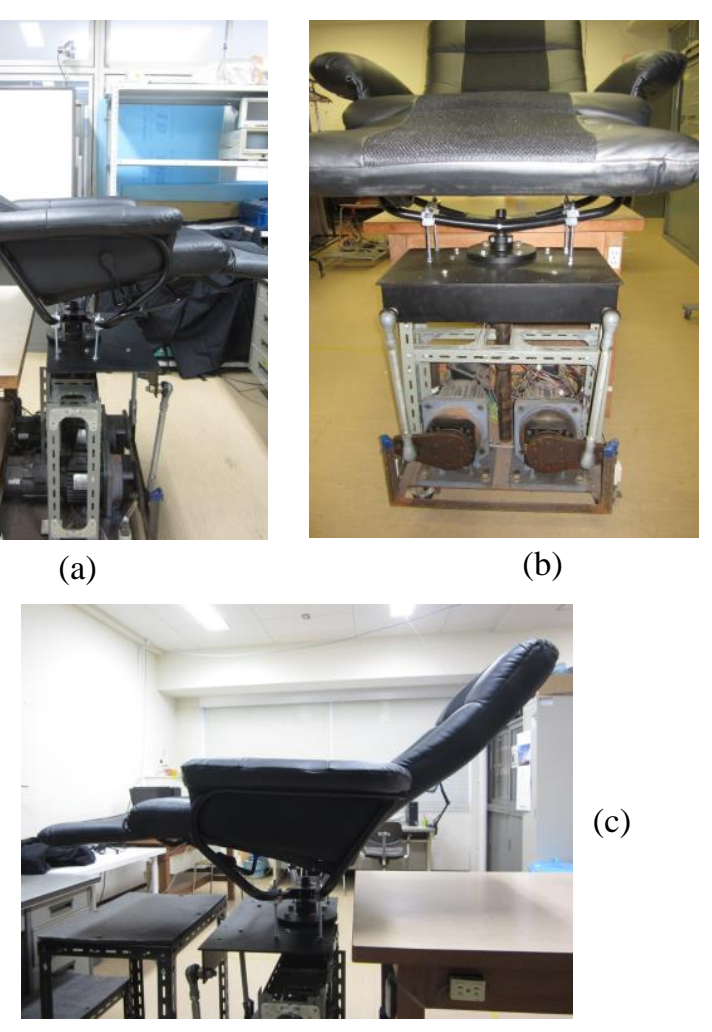

(c)

Fig.2 Prototype of SWS (a) Appearance

(b) Side view (c) Front view

\subsection{Control system}

Figure. 5 shows a configuration of a control system of SWS. The control system consists of servo motors, a motor drive unit, and a wave generator. First, the wave generator computes trajectories planning for the SWS's seat. Computed target trajectories are divided into 2 axis position information for each servo motor. After that, values of 2 axis 
position information are sent from the wave generator to the servo motor drive unit at every $25 \mathrm{~ms}$. The motor drive unit controls 2 servo motors to trace the received position information values accurately. The servo motors and their drive unit configure servomechanism for accurate position control. This is the way to realize the function of creating sea waves in the seat.

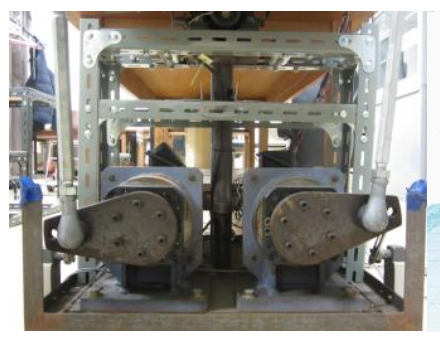

(a)

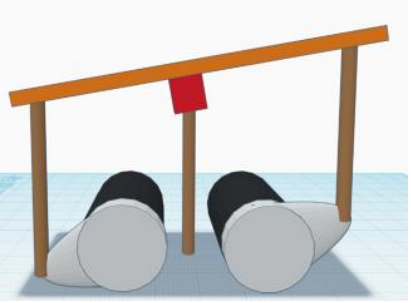

(b)
Fig.3 Front view of SWS tilted leftward

(a) Appearance (b) Drawing on CAD

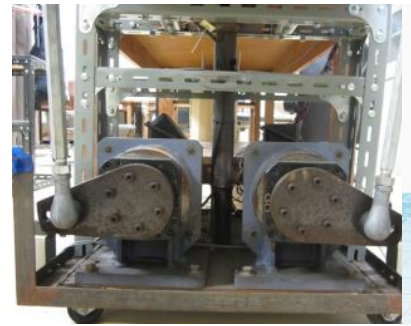

(a)

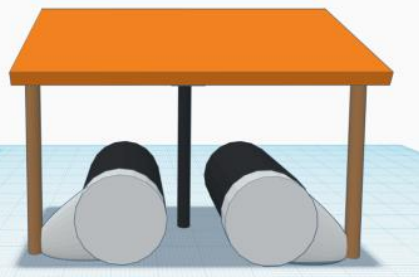

(b)
Fig.4 Front view of SWS tilted frontward

(a) Appearance (b) Drawing on CAD

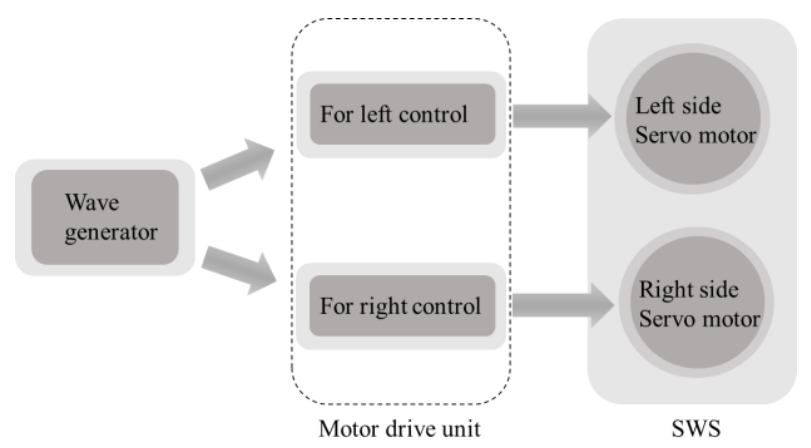

Fig.5 Configuration of designed SWS control system

\subsection{Indices extracted from HRV}

In this study, four types in the way of physiological indexes, such as LF/HF, ellipse area, SD1/SD2, and SD1, are adopted to assess the comfort level. The spectral analysis applied FFT method in HRV is widely used for human state evaluation. Value in a period from 0.04 to $0.15 \mathrm{~Hz}$ is called a low-frequency (LF) component, and from 0.15 to $0.40 \mathrm{~Hz}$ is called a high-frequency (HF) component. Parasympathetic nerve activity contributes to both LF and HF, whereas sympathetic nerve activity only affects LF. LF/HF widely used as a criterion of sympathetic nerve activity is employed as one of the evaluation indices in this study. Lorentz plot (LP) known as an evaluation method for HRV is actively researched in recent years [12-15]. The peak of the heartbeat waveform is called R-wave, and the space between R-waves is called R-R interval (RRI). Fluctuation of RRI is called HRV which is known to get influenced by both sympathetic and parasympathetic nerve activities. The LP is a 'scatterplot' of the current $\mathrm{R}-\mathrm{R}$ interval against the $\mathrm{R}-\mathrm{R}$ interval immediately preceding it, as illustrated in Fig.6. In a healthy person, LP tends to look compact when humans are active because HRV decreases by sympathetic nerve activation. Whereas the data scatter on the LP under inactive state because HRV increases by parasympathetic nerve activation. Several ways of evaluation with LP for the human state have been proposed in the fields of many. Project all the plotted data on the LP onto $y=x$ axis called line-of-identity, and $y=$ $-x$ axis. The standard deviations (SD) on the $y=-x$ axis and $\mathrm{y}=\mathrm{x}$ axis are called SD1 and SD2 respectively. The ellipse area is expressed as follows.

$$
\mathrm{S}=\pi \times \mathrm{SD} 1 \times \mathrm{SD} 2
$$

Evaluation method of parasympathetic nerve activity using this value is widely used [12]. SD1/SD2 ratio between the major axis and minor axis is also frequently employed as a criterion of sympathetic nerve activity [13]. Since there are lots of reports that SD1 is strongly correlated to parasympathetic nerve activity, we use this one as one of the parameters [14,15]. In this study, we employ these 4 parameters to evaluate the human state.

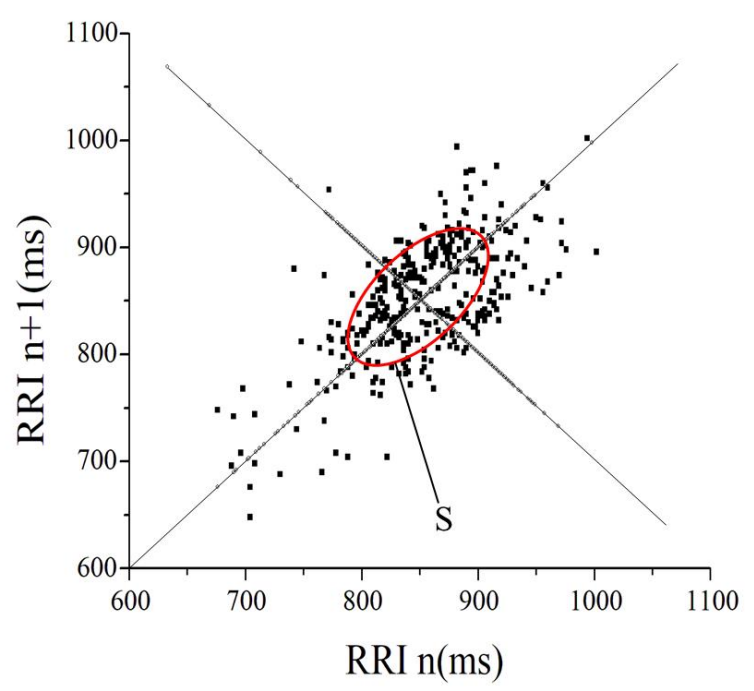

Fig.6 Evaluation method on Lorentz plot 
Question : How comfortable did you feel on SWS?

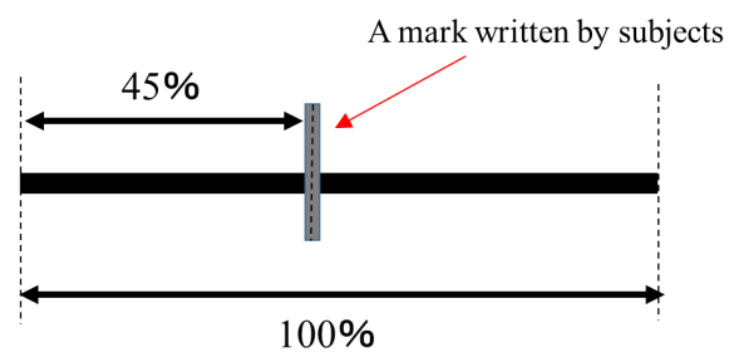

Fig.7 Appearance of VAS used in this experiment

\subsection{Visual analog scale}

Generally, there are four kinds of scales called nominal scale, ordinal scale, interval scale, and ratio scale. The ordinal scale represents the difference in both the objects and the interval scale assumes equal intervals of both the difference. The ratio scale is able to be treated as numeric values. Likert scale (LS) being a general method is thought of as the ordinal scale. Since the visual analog scale (VAS) is capable of the interval scale, this method is used for statistical analysis as well. It is one characteristic of VAS that it easily obtains subjective human feeling as intuitive values better than LS having the function of evaluation in 5 or 7 steps. The evaluation of LS has a tendency to lead to an appearance of the halo effect, generosity effect, and centralization tendency. In the case of 5 steps evaluation, for example, the centralization tendency is observed at 1 or 5 as the extreme values, and the evaluation results tend to be 3 as the center value among the numbers. In contrast, VAS in the way of representing the value in a number line ranging from 0 to 100 percent is possible to evaluate human's sensitivity on the sensory scale instead of the order scale, so that we are able to examine various statistical analysis (Fig.7). The details of VAS as a subjective evaluation method are previously reported in [16]. For the above reasons, we utilize VAS as the criterion for subjective evaluations in this study.

\subsection{Experimental procedure}

In this study, the evaluation of comfort on SWS is examined from the viewpoint of both subjective and objective evaluations. The experiment is performed with the following procedure. Subjects wearing a heartbeat sensor on their chest ride on the seat of SWS. We use a heartbeat sensor WHS-1 and analyzing software manufactured by Union Tool Inc. The subject performs simple mental arithmetic for 30 seconds, and the measurement starts subsequently. In the case of the "active" mode of SWS, a swinging operating is started when the measurement is started simultaneously. A measurement time in one experiment is 3 minutes. The information of heart rate during the experiment is transmitted to the analyzing computer via Bluetooth and recorded. After the experiment, the subjects answer the question about their feeling using VAS. We evaluate the subjective comfort level of SWS based on these data.

\section{Result and discussion}

In order to evaluate the usefulness of SWS, we conducted experiments in 3 cases; "ordinary" of ordinary chair, "inactive" mode of SWS, and "active" mode of SWS. The ordinary chair is named "ordinary" which doesn't provide any wave. The "active" mode produces waves like sea waves and the "inactive" mode doesn't provide it. The data were obtained on 28 times experiments with eleven healthy subjects aged between 19 and 23 years. All the data were first visually inspected and then processed using a threshold-based artifact correction algorithm. Measured data in 3 cases were compared and considered using four types of evaluation indices mentioned above. Table. 1 shows the average values of all data on the ellipse area, SD1/SD2, SD1, and $\mathrm{LF} / \mathrm{HF}$. The values of the ellipse area for each VAS are shown in Fig.8. The vertical axis values were divided by 1000 to scale down. The average values were 3.65 for "ordinary", 3.72 for "inactive", and 3.67 for "active", respectively. With "ordinary" as a baseline, two sample Ttests on "inactive" and "active" were performed to compare P-values. As the result, it did not achieve statistical significance because all $\mathrm{P}$-values were $\mathrm{P}>0.05$. Parasympathetic nerve activity in three cases was approximately the same level which means that their psychological state did not affect parasympathetic nerve activity. Figure. 9 shows the results of SD1/SD2 for each VAS data. The average values were 0.33 for "ordinary", 0.46 for "inactive", and 0.45 for "active", respectively. P-values of "inactive" and "active" based on "ordinary" were both statistically significant differences with $\mathrm{P}<0.01$. In comparison between "inactive" and "active", there was no significant difference with $\mathrm{P}>0.05$. The difference of sympathetic nerve activity was confirmed between SWS and ordinary chair, then sympathetic nerve activity on SWS had been suppressed lower than that of the ordinary chair. This means that sympathetic nerve activity was kept low under the state of psychological comfort. 


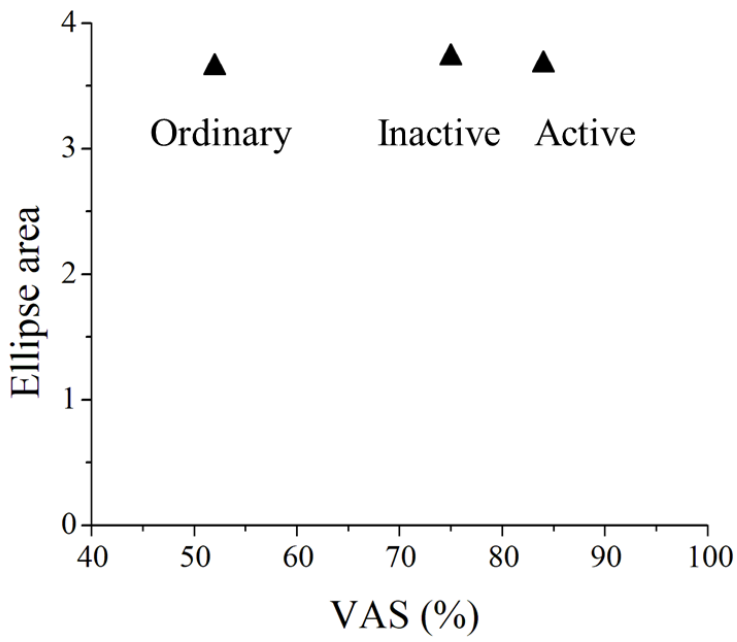

Fig.8 Comparison of the results of Ellipse area in respective VAS data

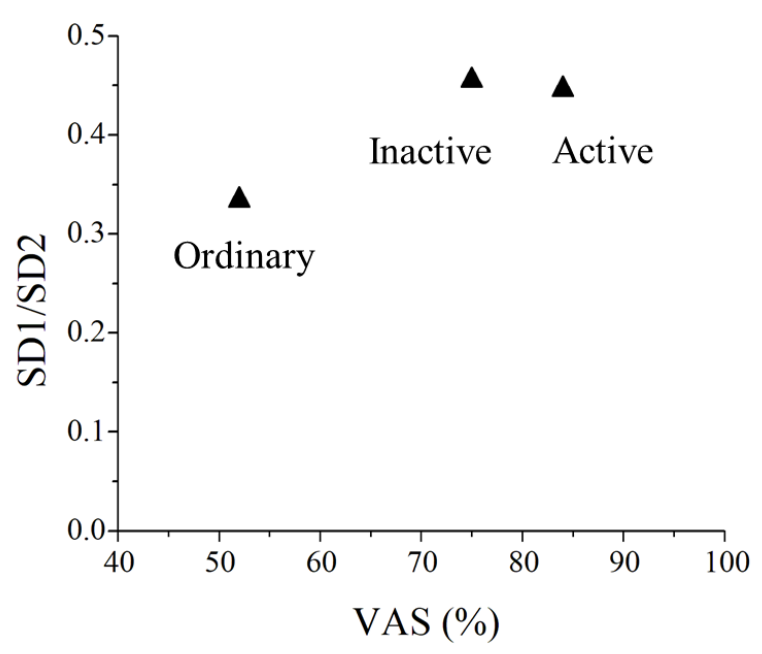

Fig.9 Comparison of the results of

$\mathrm{SD} 1 / \mathrm{SD} 2$ in respective VAS data

Table. 1 The average value of each data of HRV

\begin{tabular}{|c|ccc|}
\hline HRV & Ordinary & Inactive & Active $<0.05{ }^{* *} \mathrm{P}<0.01$ \\
\hline Area & 3647.31 & 3724.8 & 3668.45 \\
SD1/SD2 & 0.33 & $0.46^{* *}$ & $0.45^{* *}$ \\
SD1 & 18.56 & 21.94 & 21.49 \\
LH/HF & 3.06 & $1.60^{*}$ & $1.49^{*}$ \\
VAS (\%) & 52.0 & $75.0^{* *}$ & $83.5^{* *}$ \\
\hline
\end{tabular}

Figure.10 shows the results of SD1 in each VAS data. The average values were 18.56 for "ordinary", 21.94 for "inactive", and 21.49 for "active", respectively. Parasympathetic nerve activities were approximately on the same level in evaluation with SD1 because all the P-values based on "ordinary" were $\mathrm{P}>0.05$.

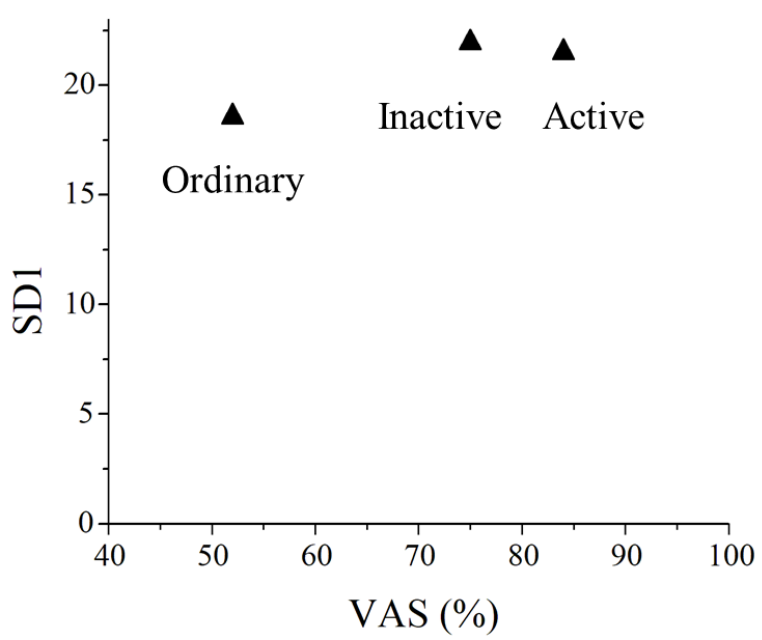

Fig.10 Comparison of the results of

SD1 in respective VAS data

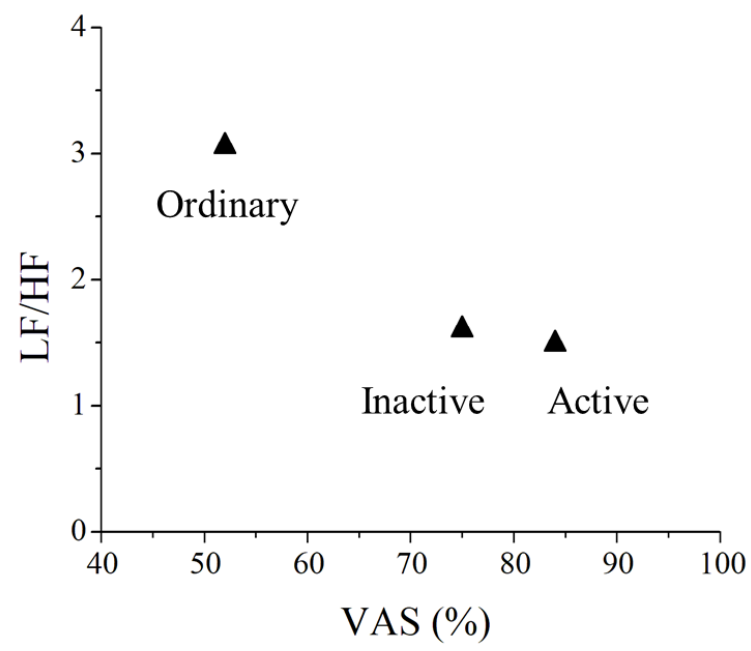

Fig.11 Comparison of the results of

$\mathrm{LF} / \mathrm{HF}$ in respective VAS data

Figure.11 shows plotted LF/HF for each VAS data. The average values were 3.06 for "ordinary", 1.60 for "inactive", and 1.49 for "active", respectively. P-values of "inactive" and "active" based on "ordinary" achieved both statistically significant differences with $\mathrm{P}<0.05$. The difference was not confirmed in comparison between "inactive" and "active" $(\mathrm{P}>0.05)$. A clear difference was confirmed between SWS and ordinary chair, then sympathetic nerve activity of SWS was suppressed lower than that of the ordinary chair. This means that sympathetic nerve activity was kept low under the state of psychological comfort. VAS data as subjective evaluations were also compared by P-values. Comparing "inactive" and "active" by P-values using "ordinary" as the baseline, both "inactive" and "active" were $P<0.01$. In comparison between "inactive" and "active" also indicated 
$\mathrm{P}<0.01$. It means that subjects had different feelings in three cases. From the above results, differences in the psychological state did not affect parasympathetic nerve activity because no significant difference was observed among the three cases. Regarding sympathetic activity, the difference between SWS and the ordinary chair was confirmed and sympathetic activity of SWS was kept lower than that of the ordinary chair. Those who psychologically comfortable tended to suppress sympathetic nerve activity. In addition, from the result of VAS, subject feelings were different in all cases, then subjects felt comfortable in order of "active" > "inactive"> "ordinary".

\section{Conclusions}

In this paper, we proposed SWS that provides a comfortable environment to release stress. This paper described the mechanical structure and control method of the developed prototype SWS. Then we conducted the experiment to assess the comfort of the SWS with subjective and objective evaluations. In order to evaluate the usefulness of SWS, we conducted experiments in 3 cases; ordinary chair, "inactive" mode of SWS, and "active" mode of SWS. In the case of parasympathetic activity, no significant difference was observed between "ordinary", "inactive", and "active". On the other hand, significant differences were observed between SWS (inactive, active) and ordinary chair in sympathetic nerve activity, and SWS suppressed it lower than the ordinary chair. In addition, from the result of VAS, the feelings of subjects were different in all cases, and people felt comfortable in order of "active" > "inactive" > "ordinary". It was found that SWS was more comfortable than the ordinary chair based on the results of both subjective and objective evaluations. Moreover, "active" was more comfortable than "inactive" on subjective evaluation. In the future, we develop a pleasant oscillation quite similar to ocean waves on SWS and then investigate its comfort level in detail from the aspect of physiological response.

\section{Acknowledgment}

Special thanks to our technical officials Ryoichi Yagami, Kazunobu Kitajima, Shoko Yamada, Tatsuo Shimosako, and Takashi Soken for their dedicated support in fabricating and adjusting the system. Furthermore, I would like to express my gratitude to all the people who participated in the experiments.

\section{References}

(1) H.Tagaya, " Measurement of Sleep-Related Hormones", Medical and Biological Engineering, Vol.46, No.2 pp.169-176, 2008.

(2) O.Troynikov, C.Watson, N.Nawaz, "Sleep environments and sleep physiology: A review", Journal of Thermal Biology 78 (2018), pp.192-203.

(3) R.Stickgold and D.Manoach, "The Importance of Sleep in Fear Conditioning and Posttraumatic Stress Disorder", Cognitive Neuroscience and Neuroimagin, March 2017, pp.109-110.

(4) Ministry of health, Labour and Welfare, National

Health and Nutrition Survey Results in 2015, https://www.mhlw.go.jp/stf/houdou/0000142359.html (accessed 2018-11-3).

(5) A.Reynolds, S.Appleton, T.Gill, A.Taylor, D.McEvoy, S.Ferguson, R.Adams, "Sickness absenteeism is associated with sleep problems independent of sleep disorders: results of the 2016 Sleep Health Foundation national survey", Journal of the National Sleep Foundation, pp.1-5, 2017.

(6) Y.Doi, "Prevalence and health impacts of sleep disorders in Japan”, J.Natl.Inst.Public Health, Vol.61, No.1, pp.3$10,2012$.

(7) K.Suzuki, "Measure comfort: evaluation of its psychological, behavioral and physiological effects", Japan Publication Service, 2002, in Japanese.

(8) T.Kawashima, "Study for Comfortable Swinging: Comfortable Swinging for Active Rocking Chair", The Japan Society of Mechanical Engineers, Vol.69, No.677, pp.219-226, 2003.

(9) T.Kawashima, "Development of Active Rocking Chair Changing the Swing with Heartbeat Fluctuation", The Japan Society of Mechanical Engineers, Vol.71, No.709, pp.2731-2737, 2005.

(10) T.KAWASHIMA, "Basic research on a semi-active incar crib with joint application of regular and inverted pendulum mechanisms", The Japan Society of Mechanical Engineers, No.15, Vol.11, pp.345-350, 2015.

(11) T.ONO, H.INOOKA, "2Degree-of -freedom Actively controlled Bed for Ambulances", The Japan Society of Mechanical Engineers, Vo.71, pp.46-52, 2005.

(12)F.Toyofuku, K.Yamaguchi, and H.Hagiwara, "Simplified method for estimating parasympathetic nervous activity by Lorenz plot of ECG RR intervals", ergonomics society, Vol.43, No.4, pp.185-192, 2007. 
(13) N.Dodo, "Effects of Sound Tempos on Autonomic Nervous System Functions”, J Psychol Sci, No.8, pp.713, 2012.

(14) S.Rahman, M.Habel, Richard J. Contrada, "Poincaré plot indices as measures of sympathetic cardiac regulation: Responses to psychological stress and associations with pre-ejection period", Int J Psychophysiol, Vol.133, pp.79-90, 2018.
(15) Peter W.KAMEN, H.KRUM and Andrew M.TONKIN, "Poincare plot of heart rate variability allows quantitative display of parasympathetic nervous activity in humans”, Clinical Science, Vol.91, pp.201-208, 1996.

(16) N.Shirahamaa, K.Murakamib, S.Watanabec, N.Nakaya and Y.Mori, "Subjective Evaluation Experiment of Grayscale Color to Examine VAS Measurement Method", Proceedings of the 6th IIAE International Conference on Intelligent Systems and Image Processing 2019, pp.147-153, 2019. 